\title{
BMJ Global Health Improving the detection of environmental enteric dysfunction: a lactulose, rhamnose assay of intestinal permeability in children aged under 5 years exposed to poor sanitation and hygiene
}

\author{
W A Faubion, ${ }^{1}$ M Camilleri, ${ }^{1} \mathrm{~J}$ A Murray, ${ }^{1}$ P Kelly, ${ }^{2}$ B Amadi, ${ }^{2}$ M N Kosek, ${ }^{3}$ \\ F Enders, ${ }^{4}$ J Larson, ${ }^{4}$ Madhusudan Grover, ${ }^{1}$ G Boe, ${ }^{1}$ R Dyer, ${ }^{5}$ R Singh ${ }^{5}$
}

To cite: Faubion WA, Camilleri M, Murray JA, et al. Improving the detection of environmental enteric dysfunction: a lactulose, rhamnose assay of intestinal permeability in children aged under 5 years exposed to poor sanitation and hygiene. $B M J$ Global Health 2016;1:e000066. doi:10.1136/bmjgh-2016000066

- Additional material is available. To view please visit the journal (http://dx.doi.org/ 10.1136/bmjgh-2016000066)

Received 18 April 2016 Revised 7 June 2016 Accepted 8 June 2016

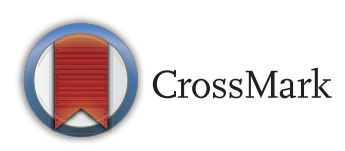

For numbered affiliations see end of article.

\section{Correspondence to}

Dr W A Faubion;

Faubion.william@mayo.edu

\section{ABSTRACT}

Background: Environmental enteric dysfunction (EED) is an asymptomatic intestinal disorder affecting populations living in conditions of poor sanitation and hygiene. The study tested intestinal barrier function in infants with EED.

Methods: We prospectively studied an advanced highperformance liquid chromatography mass spectrometry assay of urine collected after oral intake of the monosaccharide, L-rhamnose and the disaccharide, lactulose, in 112 children from three continents.

Findings: Compared to the US cohort $(n=27)$, the cohorts of children from Peru $(n=19)$ and Zambia $(n=85)$ were older with evidence of growth impairment. The median (range) of age (months) was 8.0 (2.0 to 13.0), 27.0 (15.0 to 29.0) and 21.0 (12.0 to 36.0), respectively. The median (range) of height for age $Z$ score was -0.1 ( -1.8 to 2.4$),-1.8(-3.3$ to -0.2$)$ and -2.3 (-8.5 to 1.2$)$, respectively. Among children with valid sugar data ( $n=22$ USA, $n=19$ Peru, $n=73$ Zambia), there were no significant differences in the median rhamnose urine concentrations between the three groups. The median (range) lactulose concentration $(\mu \mathrm{g} / \mathrm{mL})$ was $6.78(0.29$ to 31.90$), 47.60$ (4.23 to 379.00 ) and 75.40 ( 0.67 to 873.00 ) in the US, Peruvian and Zambian cohorts, respectively $(p<0.001)$. The lactulose/rhamnose ratio (LRR) was higher in cohorts from Peru $(0.75,0.15,5.02)$ and Zambia $(2.26,0.08$, $14.48)$ compared to the US $(0.14,0.06,1.00)$ cohort $(p<0.001)$. In a multivariate effect modification model, higher weight-for-age z scores were associated with lower post-dose lactulose when rhamnose excretion was constant $(p=0.003)$.

Conclusions: This non-invasive two saccharide permeability protocol measures changes in intestinal permeability in children with EED and permits the identification of individuals for interventional trials.

\section{INTRODUCTION}

Environmental enteric dysfunction (EED) is a disorder of the small bowel affecting

\section{Key questions}

What is already known about this topic?

- Environmental enteric dysfunction (EED) is a disorder of the small bowel affecting populations living in conditions of poor sanitation and hygiene.

- EED leads to chronic malnutrition and stunting, which affects $26 \%$ of children $<5$ years of age in the developing world and leads to $21 \%$ of deaths in this age group.

- There is a critical need for a reliable, simple, non-invasive and sensitive biomarker of disease activity to potentially identify in the field highrisk individuals who are candidates for interventional trials.

- The current application of the lactulose/mannitol assay inconsistently identifies at-risk or affected patients from endemic populations.

What are the new findings?

- We demonstrate a novel method that refines the saccharide intestinal permeability assay through the choice of monosaccharide, the method of detection, the timing of urine collection and the dose of analytes.

- We provide normative values of the lactulose/ rhamnose permeability assay in healthy infants.

- We document evidence for increased intestinal permeability in two cohorts of children from populations in which EED is endemic.

- The method described has the potential to provide consistent test performance in the field for the detection of impaired intestinal barrier integrity in EED and selection of patients for therapeutic trials.

populations living in conditions of poor sanitation and hygiene. ${ }^{1}$ It is hypothesised that repetitive intestinal infection results in chronic intestinal inflammation, villous 


\section{Key questions}

\section{Recommendations for policy}

- We recommend the use of rhamnose over mannitol when performing the saccharide intestinal permeability assay given the significant environmental contamination of the latter.

- We recommend the reporting of both test analytes (over simply a ratio), as saccharide intestinal absorption reflects complex intestinal physiology related to both permeability and loss of surface area.

- When using high-performance liquid chromatography with tandem mass spectrometry as the saccharide quantification methodology, a shortened 60-90 min urine collection window may lead to a more focused evaluation of small bowel permeability and enhanced test performance.

atrophy, malabsorption and linear growth failure. EED is a putative underlying cause of chronic malnutrition and stunting, which affects $26 \%$ of children under 5 years of age in the developing world and is associated with $21 \%$ of deaths in this age group (http://www.un.org/ millenniumgoals). Poor nutrition is associated with cognitive stunting with implications for future socioeconomic status. ${ }^{2}$ EED may also explain the reduced efficacy of oral vaccines in low income and middle income countries and the increased risk of serious infection in children with undernutrition. ${ }^{34}$ Thus, there is a critical need for a reliable, simple, non-invasive and sensitive biomarker of disease activity to potentially identify in the field high-risk individuals who are candidates for interventional trials.

Saccharide-based permeability assays (eg, lactulose/ mannitol) have been widely used in malabsorptive conditions; however, the current application of the lactulose/ mannitol assay inconsistently identifies at-risk or affected patients from endemic populations. ${ }^{5}$ Potential limitations in test performance include the enzymatic method to measure the saccharides, the timing of urine collection and the test saccharides chosen. The standard 5-hour urine collection period has led to the unclear relationship between probe concentration and permeability of different gastrointestinal (GI) tract segments. Finally, since mannitol was identified at baseline (before oral administration of the test sugar) among adults studied in the USA in up to $30 \%$ of participants, ${ }^{6}$ significant concerns exist regarding contributions to the analyte in the collected sample by the same saccharide from non-probe sources. The monosaccharide L-rhamnose is a hydrophilic deoxy sugar of similar molecular weight and volume (by van der Walls surface) as mannitol, but it is less widely used in food and other materials and is therefore an alternative candidate for evaluation of malabsorption and intestinal permeability.

Our study aim was to address the principal limitations of the current LM test and prospectively compare intestinal barrier function in infants with EED from at-risk populations on two continents (age below 37 months) with healthy control infants (2-13 months of age) from the USA. We describe a standardised method including administration protocol, consistent urine collection applicable at points of care in the field, and a reliable assay that can be performed at a centralised laboratory using liquid chromatography-mass spectrometry. The sensitivity and accuracy of this analytical chemistry approach has allowed our laboratory to reduce (by a factor of 5) the administered doses of rhamnose and lactulose, a change that both makes the test easier to tolerate and more likely to proceed without confounding by major alterations of intestinal motility and transit. ${ }^{7}$ Furthermore, by shortening the time of urine collection, the permeability deficits documented are more likely a result of permeability in the small bowel where EED changes are thought to occur and also make the test easier to perform in the resource limited settings.

\section{METHODS}

\section{Participants}

Olmsted County, MN: Healthy infants between 2 and 13 months of age $(n=27)$ were recruited from the outpatient practice at Mayo Clinic, Olmsted County, Minnesota, USA. These children were recruited at wellchild visits during routine preventative care $(2,4,6,9$ and 12 months). Age (months), length $(\mathrm{cm})$ and weight $(\mathrm{kg})$ were recorded. Additional metadata recorded included the time of last oral intake, current medications or symptoms within previous 48 hours to the study visit.

Lusaka, Zambia: Children between 2 and 36 months $(n=85)$ were recruited from one sector of the Misisi township in Lusaka, the capital of Zambia. Misisi compound is a district of southern Lusaka in which studies of EED have been conducted since $1999 .^{8}$

Loreto, Peru: A second cohort $(n=19)$ of patients from an EED region (age range 15-29 months) was recruited from three rural communities in the Department of Loreto, $15 \mathrm{~km}$ southeast of Iquitos, Peru. Studies of EED have been performed there since $2009 .^{9}$

\section{Eligibility criteria}

Children with concurrent illness or who had had diarrhoea within 1 month, or who had been given antibiotics within 1 month were excluded from study. Patients with a positive stool sample for ova and parasites at least 7 days prior to the study received treatment prior to the saccharide assay.

\section{Ethical committee approval}

Approval for the study was obtained from the University of Zambia Biomedical Research Ethics Committee (006-01-13, 11 April 2013) and the Institutional Review Board of the John's Hopkins Bloomberg School of Public Health and the Ethics Committee of Asociacion Benefica PRISMA in Lima, Peru. ${ }^{9}$ The study was approved by the Mayo Clinic Institutional Review Board (12-003757, approved 18 April 2012), and informed consent was obtained by the parent(s) or legal guardian(s). 


\section{Urine collection protocol}

The clinical protocol including saccharide administration, timing of urine collection and anthropometric data collection was identical across the three sites. The reagents were purchased from TCI chemicals, Portland, Oregon (USA and Peru) and Sigma-Aldrich, St Louis, Missouri, USA (Zambia). Aliquots of frozen urine were shipped on dry ice to the ICL at Mayo Clinic. Urine samples were collected pre dosing and post dosing.

\section{Administration of sugars}

The oral solution consisted of $200 \mathrm{mg}$ monosaccharide (L-rhamnose, TCI product code R0013, Portland, Oregon, USA) and $1000 \mathrm{mg}$ disaccharide (lactulose, TCI product code L0140 Portland, Oregon, USA) in $10 \mathrm{~mL}$ sterile water. Study personnel administered the entire solution over a period of $5 \mathrm{~min}$ to the infant. Annotations were collected for notable issues during dosing (infant spitting up, refusal, etc).

\section{Post-dose urine collection}

The urine bag was placed on the infant $30 \mathrm{~min}$ post dosing and collected over the subsequent 60 min (3090 min post dosing). Oral intake (water, breast milk, formula, no solid foods) after administration of sugar solution was encouraged with a goal of $4 \mathrm{~mL} / \mathrm{kg}$ intake over $30 \mathrm{~min}$ to promote urine production. If no urine was present at the end of $60 \mathrm{~min}$, the collection was continued until urine was present in the bag but no longer than an additional 30 min.

Samples were labelled with participant study ID and with a pre-dose or post-dose tag. Samples were stored at $-20^{\circ} \mathrm{C}$ until analysis. Batched samples were placed on dry ice and delivered to the Immunochemical Core Laboratory (ICL) at Mayo Clinic for analysis.

\section{Methodology of HPLC MS:MS}

The methodology was performed as recently published with the following modifications to account for the molecular weight transition for rhamnose. ${ }^{6}$ The following transitions were monitored: mannitol analyte (181.05/89), ${ }^{13} \mathrm{C}(6)$ mannitol IS (186.9/60.9), lactulose analyte $(341 / 101){ }^{13} \mathrm{C}(12)$ lactulose I.S. (353/167), rhamnose analyte $(163 / 58.8)$. The rhamnose analyte was quantified using the mannitol I.S. Mannitol, lactulose and rhamnose were used to make the standard curves and the sources for the internal standard were purchased from Sigma-Aldrich (St. Louis, Missouri, USA). Analytical validation of the assay is included in online supplementary materials.

\section{Statistical analysis}

Patient characteristics within each population were summarised using the median and range for continuous measures. Categorical measures were summarised as counts and percentages. Fractional recovery of rhamnose and lactulose levels across the three cohorts was assessed using the Kruskal-Wallis test. Post-dosing concentrations of rhamnose and lactulose as well as the lactulose: rhamnose ratio (LRR) and anthropomorphic measures were compared in the three groups using the Kruskal-Wallis test. Detectable urinary rhamnose (concentration $>1.0 \mu \mathrm{g} / \mathrm{mL}$ ) in the post dosing urine specimen was required to consider the test evaluable. The 95th centile of the LRR in the US cohort was defined a priori as the maximum for a normal, healthy population.

Weight-for-age $\mathrm{Z}$ scores (WAZ) and height-for-age $\mathrm{Z}$ scores (HAZ) were compared with post-dose lactulose in scatterplots showing the study cohort (symbol) and quartile of post-dose rhamnose (colour). Linear regression was used to predict WAZ scores and HAZ scores with post-dose lactulose, post-dose rhamnose and the interaction of these two predictors in two separate models. All analyses were performed using the SAS software (V.9.4, SAS Institute, Cary, North Carolina, USA).

\section{Sample size calculation}

The coefficient of variation for the assay of Mannitol and Lactulose in adult healthy participants is $33 \%$ and $50 \%$, respectively. Detectable difference with $\mathrm{n}=12$ per group (eg, healthy vs EED) would have $80 \%$ power to detect a difference in rhamnose excretion of $40 \%$ and in lactulose excretion of $58 \%$. These predictions were based on a $>600 \%$ increase in L:M ratio in Crohn's disease $(0.054 \pm 0.060$ in health vs $0.323 \pm 0.253$ in Crohn's disease).$^{10}$ Furthermore, in irritable bowel syndrome (IBS), which is typically associated with diarrhoea, there is at least a twofold increase in small intestinal permeability relative to controls and IBS associated with constipation; small intestinal permeability was increased in IBS $(0.19(0.12-0.23))$ in contrast to constipated IBS (0.085 $(0.043-0.13))$ and controls $(0.07(0.035-0.19)) .^{11}$

\section{RESULTS \\ Subject demographics}

The age and anthropometric data including median height/length and weight-for-age z-scores (HAZ and WAZ, respectively) of the three cohorts are included in table 1. Children from Peru $(n=19)$ and Zambia $(n=85)$ were older when compared to the US healthy population $(\mathrm{n}=27)$. Median (range) $\mathrm{WAZ}$ and HAZ in the Peruvian cohort (WAZ: -0.8 (-2.7, 0.8); HAZ: -1.8 $(-3.3,-0.2))$ and the Zambian cohort (WAZ: -1.4 $(-4.0,1.1)$; HAZ: $-2.3(-8.5,1.2))$ were significantly different from that in the US cohort (WAZ: $0.5(-0.4,2.1)$, $\mathrm{p}<0.001$; HAZ: $-0.1(-1.8,2.4), \mathrm{p}<0.001$, table 1$)$. Thus, while the cohorts of children from regions endemic for EED were not overtly ill, anthropometric indices suggested that they were representative of affected children with EED in these populations.

\section{Feasibility of urine saccharide absorption test}

The infants from all three cohorts followed the standard protocol of sugar administration (see methods). Evaluable tests were seen in 22/27 US infants $(81 \%)$, 
Table 1 Demographic characteristics for the study cohorts from the USA, Peru and Zambia

\begin{tabular}{lccc}
\hline & USA $(\mathbf{N}=\mathbf{2 7})$ & Peru $(\mathbf{N}=19)$ & Zambia $^{*}(\mathbf{N}=\mathbf{8 5})$ \\
\hline Age (months), median (range) & $8.0(2.0$ to 13.0$)$ & $27.0(15.0$ to 29.0$)$ & $21.0(12.0$ to 36.0$)$ \\
Gender, N (\%) & & & \\
$\quad$ F & $8(30)$ & $8(42)$ & $45(63)$ \\
$\quad$ M & $19(70)$ & $11(58)$ & $27(38)$ \\
Height-for-age z-score, median (range) & $-0.1(-1.8$ to 2.4$)$ & $-1.8(-3.3$ to -0.2$)$ & $-2.3(-8.5$ to 1.2$)$ \\
Weight-for-age z-score, median (range) & $0.5(-0.4$ to 2.1$)$ & $-0.8(-2.7$ to 0.8$)$ & $-1.4(-4.0$ to 1.1$)$ \\
$\delta$ Rhamnose $<1 \mathrm{~N}(\%)$ & $5(19)$ & $0(0)$ & $12(14)$ \\
\hline
\end{tabular}

${ }^{*}$ In the Zambian cohort, age was available on 73 participants while gender and z-scores were available on 72 participants.

19/19 Peruvian children (100\%) and 73/85 Zambian children $(86 \%)$. In the US cohort, regurgitation of saccharide solution or urine collection bag failure (leak) was the primary cause of test failure ( 5 cases compared to 22 successful test applications). Similar metadata was not available from Zambia to determine the primary causes of test failure. The median (range) of total urine volume collected $(\mathrm{mL})$ was $13.4(1.3,65.1), 21.5$ (7.0, $67.0)$ and $5.0(0.5,10.0)$ in the US, Peruvian and Zambian cohorts, respectively.

\section{Baseline urinary levels of mannitol}

Detectable concentrations of mannitol were present in over $90 \%$ of children in all three cohorts within the baseline urine sample. Since these samples were collected prior to saccharide dosing (and mannitol was not used as a probe), this finding indicates significant environmental contamination. The median (range) of the mannitol concentration identified prior to saccharide dosing in the cohorts from the USA, Peru and Zambia was $5.2 \mu \mathrm{g} / \mathrm{mL}(0.1,17.3), 10.8 \mu \mathrm{g} / \mathrm{mL}(0.0,29.7)$ and $10.3 \mu \mathrm{g} / \mathrm{mL} \quad(0.8,40.2)$, respectively (table 2). We assessed pre-dosed (baseline) urine samples for the presence of L-rhamnose. In all three cohorts, the presence of L-rhamnose in pre-administration urine was negligible (table 2). Indeed, there were only 3 individuals $(2.6 \%)$ in the entire cohort who had pre-dosing urine levels of rhamnose and lactulose that theoretically could interfere with the interpretation of the assay results.

\section{Permeability measurements using fractional excretion of saccharides and lactulose: rhamnose ratio in participants at risk for EED}

Rhamnose excretion: There were no significant differences in the permeability to rhamnose as assessed by median urinary rhamnose concentrations between the three groups (table 3, figure 1). The fractional recovery of rhamnose was significantly higher in the US $(0.38 \%$ $(0.00 \%, 1.84 \%))$ and Peruvian $(0.61 \%(0.02 \%, 6.32 \%))$ cohorts compared to the Zambian $(0.07 \% \quad(0.00 \%$, $1.50 \%)$ ) cohort $(\mathrm{p}<0.001)$ (table 3$)$.

Lactulose excretion: Significant differences in lactulose as expressed by both urine concentrations and fractional excretion were evident in the cohorts of patients from areas endemic for EED compared to the US controls (table 3, figure 1). Consistent with the lack of significant pre-dosing saccharide contamination (table 2), results were identical on analysis of $\delta$ lactulose (postdose lactulose less pre-dose concentration, data not shown). Similar to the lactulose concentration, the fractional recovery of lactulose was significantly greater in the Zambian $(0.03 \%(0.00 \%, 0.35 \%))$ and Peruvian $(0.15 \%(0.01 \%, 0.69 \%)$ cohorts when compared to the US $(0.01 \%(0.00 \%, 0.07 \%))$ cohort $(\mathrm{p}<0.001$, table 3$)$.

Lactulose to rhamnose ratio: Given the previous experience with saccharide ratios and the challenges of urine bag collection with precise urine volume recording in the field, we calculated the lactulose to rhamnose ratio based on concentrations of the sugars (LRR). We observed significant differences in the median (range) LRR in the Peruvian $(0.75(0.15,5.02))$ and Zambian $(2.26(0.08,14.48))$ cohorts compared to the US $(0.14$ $(0.06,1.00))$ cohort $(\mathrm{p}<0.001)$ (table 3 , figure 1$)$. Using the 95th centile from the US population as the upper limit of normal, $~ 53 \%$ of Peruvian children and $82 \%$ of Zambian children studied displayed abnormal LRR $(\mathrm{p}<0.001$, table 3$)$.

\section{Appraisal of height and weight relative to LRR}

Using the HAZ and WAZ scores of children at the time of LRR within the Zambian and Peruvian cohorts, we assessed the ability of WAZ and HAZ to predict

Table 2 Median (range) of pre-dosing sugar concentrations by study cohort

\begin{tabular}{lllll}
\hline & USA $(\mathbf{N}=20)^{*}$ & Peru (N=19) & Zambia (N=63) & p Value† \\
\hline Pre-mannitol $(\mu \mathrm{g} / \mathrm{mL})$ & $5.2(0.1$ to 17.3$)$ & $10.8(0.0$ to 29.7$)$ & $10.3(0.8$ to 40.2$)$ & 0.046 \\
Pre-rhamnose $(\mu \mathrm{g} / \mathrm{mL})$ & $0.0(0.0$ to 0.4$)$ & $0.0(0.0$ to 20.1$)$ & $0.1(0.0$ to 36.9$)$ & $0.1(0.0$ to 86.1$)$ \\
Pre-lactulose $(\mu \mathrm{g} / \mathrm{mL})$ & $0.0(0.0$ to 4.0$)$ & $0.5(0.0$ to 19.9$)$ & 0.043 \\
\hline
\end{tabular}

*Pre-dosing sugar data for the U.S. cohort was available for 20 of the 27 participants and for the Zambian cohort in 63 of the 85 participants. †Kruskal-Wallis test. 
Table 3 Summary of sugar excretion following oral administration by study cohort

\begin{tabular}{|c|c|c|c|c|}
\hline & \multicolumn{4}{|c|}{ Summary of sugar excretion following oral administration by population } \\
\hline & USA (N=22) & Peru* $(\mathrm{N}=19)$ & Zambia† ( $\mathrm{N}=73)$ & p Value \\
\hline Post-rhamnose (ug/mL), median (range) & $50.10(0.29$ to 213.00$)$ & $102.00(2.66$ to 301.00$)$ & $34.50(1.58$ to 847.00$)$ & $0.354 \ddagger$ \\
\hline Post-lactulose $(\mu \mathrm{g} / \mathrm{mL})$, median (range) & $6.78(0.29$ to 31.90$)$ & 47.60 (4.23 to 379.00$)$ & $75.40(0.67$ to 873.00$)$ & $<0.001 \ddagger$ \\
\hline LR ratio, median (range) & $0.14(0.06$ to 1.00$)$ & $0.75(0.15$ to 5.02$)$ & $2.26(0.08$ to 14.48$)$ & $<0.001 \ddagger$ \\
\hline $\begin{array}{l}\text { Abnormal post LRR compared to the } \\
\text { American 95th centile }\end{array}$ & $1(5 \%)$ & $10(53 \%)$ & $60(82 \%)$ & $<0.001 \S$ \\
\hline
\end{tabular}

*Fractional recovery was available in 18 of the 19 Peruvian participants.

†Fractional recovery was only available in 67 of the Zambian participants. Other post-dose sugar concentrations were available for 73 of the Zambian participants.

$\ddagger$ Kruskal-Wallis test.

$\S \chi^{2}$ Test.

abnormal LRR as defined by $>95 \%$ centile in the US cohort (figure 2). In a multivariate effect modification model in which the outcome was WAZ score (table 4), lower post-dose lactulose was associated with higher WAZ scores among children with the same level of post-dose rhamnose $(p=0.0002)$. When children differed in rhamnose, however, those with both higher rhamnose and higher lactulose also had slightly greater WAZ scores $(p=0.01)$ after accounting for the association between lactulose and weight for age when rhamnose was the same. In this model, $13 \%$ of the WAZ score variability among children was explained by lactulose and rhamnose excretion. A similar pattern emerged when predicting height-for-age $\mathrm{Z}$ scores, but the differences were not statistically significant.

\section{DISCUSSION}

EED results from the chronic inflammatory response to repetitive GI infectious insults, and thus populations living in poverty in the developing world are commonly affected to varying degrees. A general association of the lactulose to mannitol ratio, particularly in relationship to impaired linear growth, has been noted among several groups; however, significant issues remain with the test's performance. ${ }^{5}$ We provide normative values of the lactulose/rhamnose permeability assay in healthy infants and document evidence for increased intestinal permeability in two cohorts of children from populations in which EED is endemic. These observations suggest that the method described has the potential to provide consistent test performance in the field for the detection of
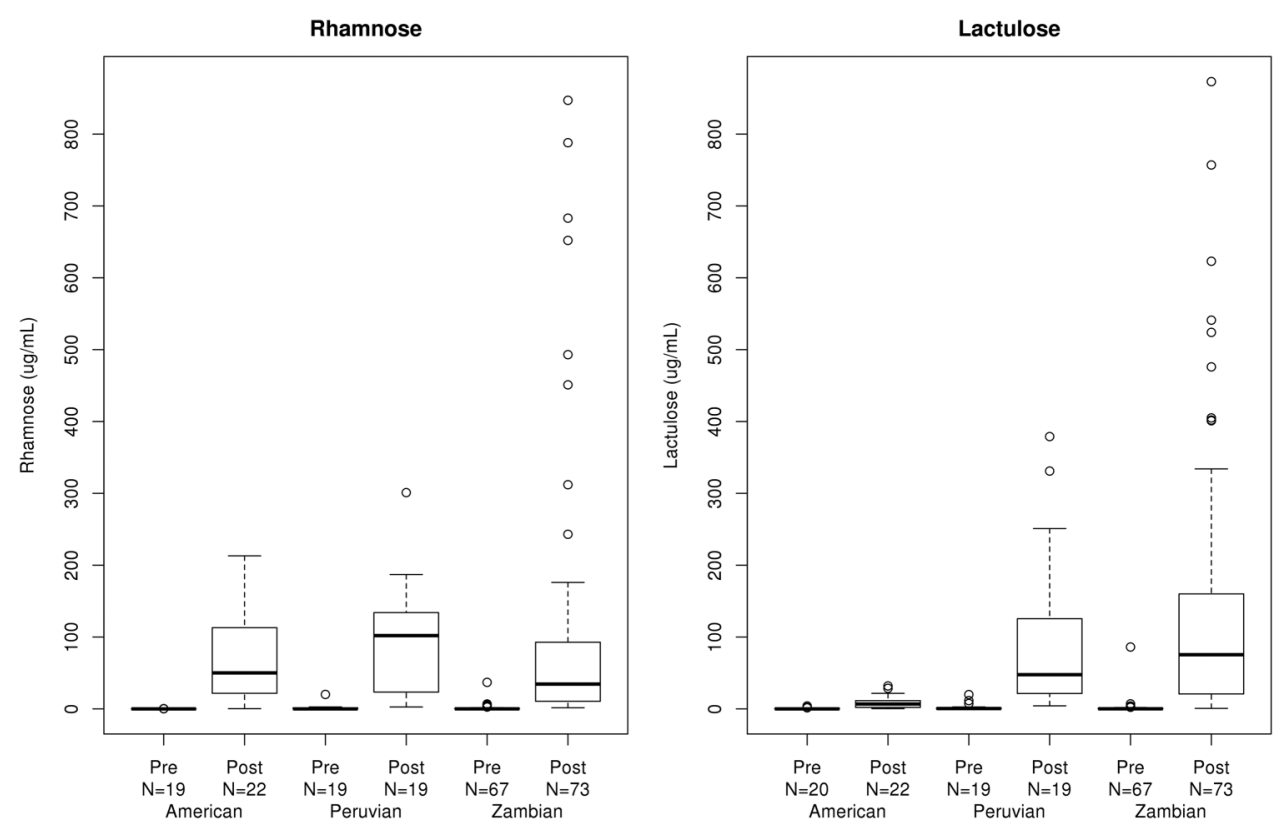

Figure 1 Box plot comparison of pre-dose and post-dose concentrations for rhamnose and lactulose for the three study cohorts. 

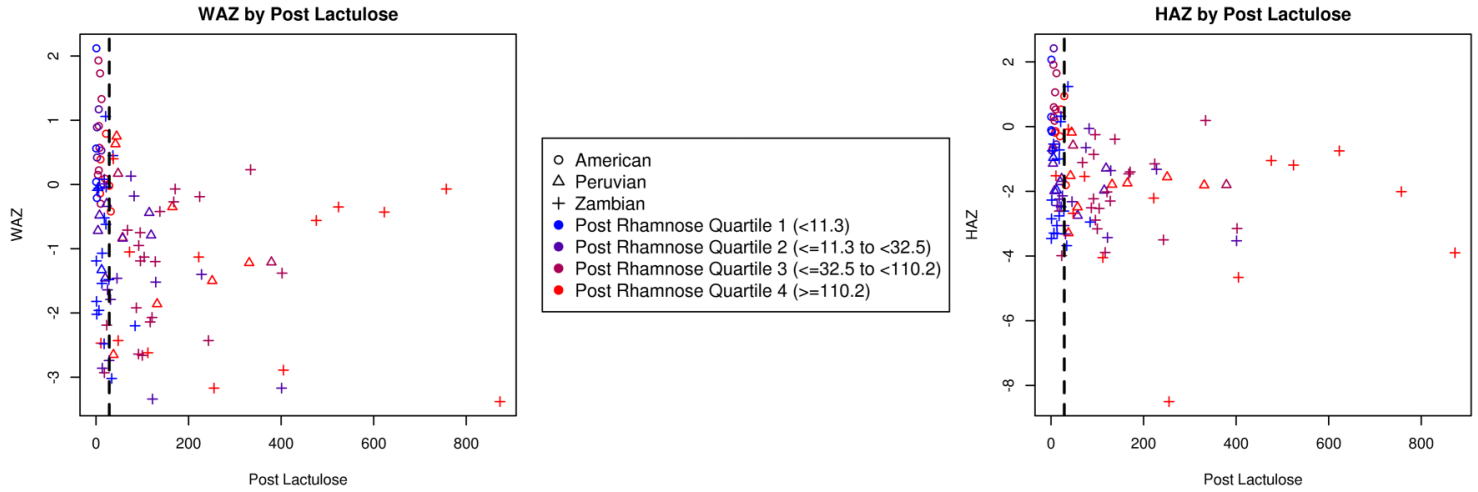

Figure 2 Scatterplot weight-for-age Z scores (WAZ) and height-for-age Z scores (HAZ) by post-dose lactulose and rhamnose concentration for the three study cohorts.

impaired intestinal barrier integrity in EED. Our data also suggest that there was monosaccharide (rhamnose) malabsorption (reduced absolute mass excreted and fractional recovery in urine) in at-risk children from Zambia compared to US controls, and that the stunted weight observed in the at-risk cohorts was associated with the documented measurements of monosaccharide malabsorption as well as increased intestinal permeability, which is best adjudicated on the basis of the lactulose excretion, fractional recovery of lactulose in urine and LRR. Thus, children in Zambia have evidence for both monosaccharide malabsorption and increased intestinal permeability to the larger disaccharide molecule.

Current understanding is consistent with the existence of two pore sizes in the human small intestinal epithelium, with the larger pore size (10.1 Angstrom) present in the crypts while the smaller pores are located in the villus tip. ${ }^{12}$ In extensive small intestinal diseases associated with significant flattening of the villus border and resultant impairment of the surface area, it is not clear whether paracellular transport through either pore is proportionally affected and the overall effect of decreased surface area is also unclear. We observed a significantly lower recovery of the monosaccharide rhamnose in the cohort of children from Zambia when compared to the US and Peruvian cohorts. Similarly, low recovery of the monosaccharide mannitol was described in affected children in Gambia, suggesting the significant contribution of malabsorption along with altered permeability in the interpretation of saccharide permeability assays in severely affected children. ${ }^{13}$ Combinatorial effects of malabsorption and altered permeability are general limitations in assessment of solute trafficking dependent on size. Indeed, the impact of mannitol excretion in driving variances in LMR across studies has been previously postulated. ${ }^{14}$ We speculate that the diminished recovery of rhamnose in the Zambian cohort reflects decreased intestinal surface area related to villus blunting and a more severe phenotype, and this may compromise the assessment of the ratio of excretion of the two saccharides in the LRR. Therefore, the absolute excretion or fractional recovery of the disaccharide alone may potentially be a more accurate marker of intestinal permeability. Overall, these data emphasise the importance of separate reporting of recovery for both saccharides.

Our data suggest the growth stunting evident in the at-risk cohorts to be associated with impaired intestinal permeability as evidenced by the coefficient of determination $\left(\mathrm{R}^{2}\right)$ of the multivariate regression model to predict weight-for-age and height-for-age $\mathrm{z}$ scores. One factor contributing to improved assay concordance may be the timing of urine collection. The simultaneous assessment of intestinal transit and lactulose and mannitol permeability in healthy adult participants indicates

Table 4 Linear regression results predicting weight-for-age and height-for-age $Z$ scores by post-dose lactulose and rhamnose concentrations and their interaction

\begin{tabular}{|c|c|c|c|c|}
\hline Outcome variable & Predictor & Coefficient $(95 \% \mathrm{CI})$ & p Value & $\mathbf{R}^{2}$ \\
\hline \multirow[t]{3}{*}{ Weight-for-age $Z$ score $(N=104)$} & Intercept & $-0.5007(-0.9248$ to 0.01766$)$ & 0.003 & $13 \%$ \\
\hline & Post lactulose & $-0.0041(-0.0063$ to -0.002$)$ & $<0.001$ & \\
\hline & Lactulose-rhamnose interaction & 0.000007 (0.00000 to 0.00001$)$ & 0.013 & \\
\hline \multirow[t]{3}{*}{ Height-for-age Z score $(\mathrm{N}=104)$} & Intercept & $-1.13512(-1.5629$ to -0.7075$)$ & $<0.001$ & $10 \%$ \\
\hline & Post rhamnose & $-0.0008(-0.0041$ to 0.0025$)$ & 0.630 & \\
\hline & Lactulose-rhamnose interaction & $0.000007(-0.00000$ to 0.00002$)$ & 0.098 & \\
\hline
\end{tabular}


that urine collection within 2 hours of oral sugar load reflects primarily small intestinal permeability while collection beyond 8 hours reflects exclusively colonic absorption. ${ }^{7}$ While equivalent data are not available in children, previous protocols have uniformly extended the collection to 5 hours during which the saccharide can reach the caecum and ascending colon in at least a subset of participants; ${ }^{15-36}$ thus, such recovery studies may have assessed both small bowel and colonic permeability with interindividual variances related to the differences in location of the saccharides during the 5 hours of urine collection.

Finally, the regression analyses show that permeability is a marker that alone accounts for $\sim 13 \%$ of the variance in WAZ. Thus, other factors such as malabsorption, recurrent infection and undernutrition constitute other risk factors that most likely contribute to the low WAZ and HAZ. Nevertheless, permeability measurement constitutes a good screening test for EED in the field, and should lead to a search for concomitant risk factors or institution of interventions to restore barrier integrity, when such drugs become available.

In conclusion, we demonstrate a novel method that refines the saccharide intestinal permeability assay. The advantages of the method that we have applied in the field in Zambia and Peru are decreased dose of saccharides administered, shortened urine collection time and use of a monosaccharide without risk of significant environmental contamination. Minimal environmental contamination may lead to the lack of reliance on baseline urine samples; yet further validation with corresponding histology in a separate disease cohort (coeliac disease) is currently ongoing.

\author{
Author affiliations \\ ${ }^{1}$ Division of Gastroenterology and Hepatology, Mayo Clinic, Rochester, \\ Minnesota, USA \\ ${ }^{2}$ Queen Mary, University of London, University of Zambia School of Medicine, \\ London, UK \\ ${ }^{3}$ Department of International Health, John's Hopkins Bloomberg School of \\ Public Health, Baltimore, Maryland, USA \\ ${ }^{4}$ Biomedical Statistics and Informatics, Mayo Clinic, Rochester, Minnesota, \\ USA \\ ${ }^{5}$ Immunochemical Core Laboratory, Mayo Clinic, Rochester, Minnesota, USA
}

Correction notice This paper has been updated since it first published online. The author, Madhusudan Grover, has been included.

\section{Handling editor Seye Abimbola}

Contributors WAF, MC, JM, PK, BA, MK and RS participated in project conceptualisation, experimental design, experimental conduct, data generation, data interpretation and manuscript preparation. FE and JL took part in experimental design, statistical support, data interpretation and manuscript preparation. $\mathrm{GB}$ and $\mathrm{RD}$ took part in data generation, experimental conduct and manuscript preparation.

Funding This project was supported by the Bill \& Melinda Gates Foundation. This publication was supported in part by Grant Number UL1TR000135 from the National Center for Advancing Translational Sciences (NCATS). Its contents are solely the responsibility of the authors and do not necessarily represent the official views of the NIH.

Competing interests None declared.
Ethics approval Approval for the study was obtained from the University of Zambia Biomedical Research Ethics Committee (006-01-13, 11 April, 2013) and the Institutional Review Board of the John's Hopkins Bloomberg School of Public Health and the Ethics Committee of Asociacion Benefica PRISMA in Lima, Peru [9]. The study was approved by the Mayo Clinic Institutional Review Board (12-003757, approved 18 April 2012), and informed consent was obtained by the parent(s) or legal guardian(s).

Provenance and peer review Not commissioned; externally peer reviewed.

Data sharing statement No additional data are available.

Open Access This is an Open Access article distributed in accordance with the Creative Commons Attribution Non Commercial (CC BY-NC 4.0) license, which permits others to distribute, remix, adapt, build upon this work noncommercially, and license their derivative works on different terms, provided the original work is properly cited and the use is non-commercial. See: http:// creativecommons.org/licenses/by-nc/4.0/

\section{REFERENCES}

1. Korpe PS, Petri WA Jr. Environmental enteropathy: critical implications of a poorly understood condition. Trends $\mathrm{Mol}$ Med 2012;18:328-36.

2. Scharf RJ, Deboer MD, Guerrant RL. Recent advances in understanding the long-term sequelae of childhood infectious diarrhea. Curr Infect Dis Rep 2014;16:408.

3. Crane RJ, Jones KD, Berkley JA. Environmental enteric dysfunction: an overview. Food Nutr Bull 2015;36(1 Suppl):S76-87.

4. Jones KD, Thitiri J, Ngari M, et al. Childhood malnutrition: toward an understanding of infections, inflammation, and antimicrobials. Food Nutr Bull 2014;35(2 Suppl):S64-70.

5. Denno DM, VanBuskirk K, Nelson ZC, et al. Use of the lactulose to mannitol ratio to evaluate childhood environmental enteric dysfunction: a systematic review. Clin Infect Dis 2014;59(Suppl 4): S213-19.

6. Grover M, Camilleri M, Hines J, et al. $13 \mathrm{C}$ mannitol as a nove biomarker for measurement of intestinal permeability. Neurogastroenterol Motil 2016.

7. Camilleri M, Nadeau A, Lamsam J, et al. Understanding measurements of intestinal permeability in healthy humans with urine lactulose and mannitol excretion. Neurogastroenterol Motil 2010;22:e15-26.

8. Amadi $B$, Imikendu M, Sakala $M$, et al. Integration of HIV care into community management of acute childhood malnutrition permits good outcomes: retrospective analysis of three years of a programme in Lusaka. PLoS ONE 2016;11:e0149218.

9. Yori PP, Lee G, Olórtegui MP, et al. Santa Clara de Nanay: the MAL-ED cohort in Peru. Clin Infect Dis 2014;59(Suppl 4):S310-16.

10. Haas V, Büning $\mathrm{C}$, Buhner $\mathrm{S}$, et al. Clinical relevance of measuring colonic permeability. Eur J Clin Invest 2009;39:139-44.

11. Dunlop SP, Hebden J, Campbell E, et al. Abnormal intestinal permeability in subgroups of diarrhea-predominant irritable bowe syndromes. Am J Gastroenterol 2006;101:1288-94.

12. Linnankoski J, Mäkelä J, Palmgren J, et al. Paracellular porosity and pore size of the human intestinal epithelium in tissue and cell culture models. J Pharm Sci 2010;99:2166-75.

13. Lunn PG. The impact of infection and nutrition on gut function and growth in childhood. Proc Nutr Soc 2000;59:147-54.

14. Kosek M, Guerrant RL, Kang G et al. Assessment of environmental enteropathy in the MAL-ED cohort study: theoretical and analytic framework. Clin Infect Dis 2014;59(Suppl 4):S239-47.

15. Campbell DI, Elia M, Lunn PG. Growth faltering in rural Gambian infants is associated with impaired small intestinal barrier function, leading to endotoxemia and systemic inflammation. J Nutr 2003;133:1332-8.

16. Campbell DI, Lunn PG, Elia M. Age-related association of small intestinal mucosal enteropathy with nutritional status in rural Gambian children. Br J Nutr 2002;88:499-505.

17. Campbell DI, McPhail G, Lunn PG, et al. Intestinal inflammation measured by fecal neopterin in Gambian children with enteropathy: association with growth failure, Giardia lamblia, and intestinal permeability. J Pediatr Gastroenterol Nutr 2004;39:153-7.

18. Campbell DI, Murch SH, Elia M, et al. Chronic T cell-mediated enteropathy in rural west African children: relationship with nutritional status and small bowel function. Pediatr Res 2003;54:306-11.

19. Filteau SM, Rollins NC, Coutsoudis A, et al. The effect of antenatal vitamin $\mathrm{A}$ and beta-carotene supplementation on gut integrity of 
infants of HIV-infected South African women. J Pediatr Gastroenterol Nutr 2001:32:464-70.

20. Goto R, Mascie-Taylor CG, Lunn PG. Impact of intestinal permeability, inflammation status and parasitic infections on infant growth faltering in rural Bangladesh. Br J Nutr 2009;101:1509-16.

21. Goto R, Mascie-Taylor CG, Lunn PG. Impact of anti-Giardia and anthelminthic treatment on infant growth and intestinal permeability in rural Bangladesh: a randomised double-blind controlled study. Trans R Soc Trop Med Hyg 2009;103:520-9.

22. Goto R, Panter-Brick C, Northrop-Clewes CA, et al. Poor intestinal permeability in mildly stunted Nepali children: associations with weaning practices and Giardia lamblia infection. Br J Nutr 2002;88:141-9.

23. Lima AA, Brito LF, Ribeiro HB, et al. Intestinal barrier function and weight gain in malnourished children taking glutamine supplemented enteral formula. J Pediatr Gastroenterol Nutr 2005;40:28-35.

24. Lima AA, Soares AM, Lima NL, et al. Effects of vitamin $A$ supplementation on intestinal barrier function, growth, total parasitic, and specific Giardia spp infections in Brazilian children: a prospective randomized, double-blind, placebo-controlled trial. $J$ Pediatr Gastroenterol Nutr 2010:50:309-15.

25. Rollins NC, Filteau SM, Coutsoudis A, et al. Feeding mode, intestinal permeability, and neopterin excretion: a longitudinal study in infants of HIV-infected South African women. J Acquir Immune Defic Syndr 2001;28:132-9.

26. Rollins NC, Filteau SM, Elson I, et al. Vitamin A supplementation of South African children with severe diarrhea: optimum timing for improving biochemical and clinical recovery and subsequent vitamin A status. Pediatr Infect Dis J 2000;19:284-9.

27. Williams EA, Elia M, Lunn PG. A double-blind, placebo-controlled, glutamine-supplementation trial in growth-faltering Gambian infants. Am J Clin Nutr 2007;86:421-7.
28. Zhang $\mathrm{Y}$, Lee B, Thompson M, et al. Lactulose-mannitol intestinal permeability test in children with diarrhea caused by rotavirus and cryptosporidium. Diarrhea Working Group, Peru. J Pediatr Gastroenterol Nutr 2000;31:16-21.

29. Chen P, Soares AM, Lima AA, et al. Association of vitamin A and zinc status with altered intestinal permeability: analyses of cohort data from northeastern Brazil. J Health Popul Nutr 2003;21: 309-15.

30. Darboe MK, Thurnham DI, Morgan G, et al. Effectiveness of an early supplementation scheme of high-dose vitamin A versus standard WHO protocol in Gambian mothers and infants: a randomised controlled trial. Lancet 2007:369:2088-96.

31. Lima NL, Soares AM, Mota RM, et al. Wasting and intestinal barrier function in children taking alanyl-glutamine-supplemented enteral formula. J Pediatr Gastroenterol Nutr 2007;44:365-74.

32. Northrop-Clewes CA, Rousham EK, Mascie-Taylor CN, et al. Anthelmintic treatment of rural Bangladeshi children: effect on host physiology, growth, and biochemical status. Am J Clin Nutr 2001;73:53-60.

33. Quadro L, Gamble MV, Vogel S, et al. Retinol and retinol-binding protein: gut integrity and circulating immunoglobulins. J Infect Dis 2000;182(Suppl 1):S97-102.

34. Rabbani GH, Teka T, Saha SK, et al. Green banana and pectin improve small intestinal permeability and reduce fluid loss in Bangladeshi children with persistent diarrhea. Dig Dis SCi 2004:49:475-84.

35. Thurnham DI, Northrop-Clewes CA, McCullough FS, et al. Innate immunity, gut integrity, and vitamin A in Gambian and Indian infants. $J$ Infect Dis 2000;182(Suppl 1):S23-8.

36. Vieira MM, Paik J, Blaner WS, et al. Carotenoids, retinol, and intestinal barrier function in children from northeastern Brazil. J Pediatr Gastroenterol Nutr 2008;47:652-9. 
Correction: Improving the detection of environmental enteric dysfunction: a lactulose, rhamnose assay of intestinal permeability in children aged under 5 years exposed to poor sanitation and hygiene

Faubion WA, Camilleri M, Murray JA, et al. Improving the detection of environmental enteric dysfunction: a lactulose, rhamnose assay of intestinal permeability in children aged under 5 years exposed to poor sanitation and hygiene. BMJ Global Health 2016;1:e00066. This article has been corrected since it was first published. The author, Madhusudan Grover, has been included. The correct author list is: Faubion WA, Camilleri M, Murray JA, Kelly P, Amadi B, Kosek MN, Enders F, Larson J, Grover M, Boe G, Dyer R, Singh R.

Open Access This is an Open Access article distributed in accordance with the Creative Commons Attribution Non Commercial (CC BY-NC 4.0) license, which permits others to distribute, remix, adapt, build upon this work non-commercially, and license their derivative works on different terms, provided the original work is properly cited and the use is non-commercial. See: http://creativecommons.org/licenses/by-nc/4.0/

BMJ Glob Health 2017;2:e000066corr1. doi:10.1136/bmjgh-2016-000066corr1 